\title{
The direct costs of overdiagnosed asthma in a longitudinal population-based study
}

Running title: Costs of overdiagnosed asthma

Bryan C. Ng${ }^{1}$; Mohsen Sadatsafavi, MD, PhD ${ }^{1,2}$; Abdollah Safari, PhD ${ }^{1,2}$; J. Mark FitzGerald, $\mathrm{MD}^{2}$; Kate M. Johnson, MSc${ }^{1}$

\author{
Affiliations: \\ ${ }^{1}$ Respiratory Evaluation Sciences Program, Collaboration for Outcomes Research and \\ Evaluation, Faculty of Pharmaceutical Sciences, University of British Columbia, Vancouver, \\ Canada \\ ${ }^{2}$ Institute for Heart and Lung Health, Department of Medicine, University of British Columbia, \\ Vancouver, Canada \\ Corresponding Author: Kate M. Johnson \\ Faculty of Pharmaceutical Sciences, University of British \\ Columbia, Vancouver Campus, \\ 2405 Wesbrook Mall \\ Vancouver, BC Canada V6T 1 Z3 \\ Phone: 604-788-8575 \\ Email: kate.johnson@alumni.ubc.ca
}

\begin{abstract}
Word Count: 262
Word Count: 2836 (excluding abstract, references, tables, appendices, and figure legends)

Number of Figures and Tables: 5
\end{abstract}




\section{ABBREVIATIONS:}

2 BC: British Columbia

3 CI: Confidence Interval

$4 \quad \mathrm{FEV}_{1}$ : Forced Expiratory Volume in 1 second

5 MPR: Medication Possession Ratio

6 SD: Standard Deviation 
7 ABSTRACT

8 Objectives: A current diagnosis of asthma cannot be objectively confirmed in many patients

9 with physician-diagnosed asthma. Estimates of resource use in overdiagnosed cases of asthma

10 are necessary to measure the burden of overdiagnosis and evaluate strategies to reduce this

11 burden. We assessed the difference in asthma-related healthcare resource use between patients

12 with a confirmed asthma diagnosis and those with asthma ruled out.

13 Design: Population-based prospective cohort study.

14 Setting: Participants were recruited through random-digit dialling of both landlines and mobile

15 phones in $\mathrm{BC}$, Canada.

16 Participants: We included 345 individuals $\geq 12$ years of age with a self-reported physician

17 diagnosis of asthma which was confirmed by a bronchodilator reversibility or methacholine

18 challenge test at the end of the 12-month follow-up.

19 Primary and secondary outcome measures: Self-reported annual asthma-related direct

20 healthcare costs (2017 Canadian dollars), outpatient physician visits, and medication use from

21 the Canadian healthcare system perspective.

22 Results: Asthma was ruled out in 86 (24.9\%) participants. Average annual asthma-related direct

23 healthcare costs for participants with confirmed asthma were \$497.9 (SD \$677.9), and \$307.7

24 (SD \$424.1) for participants with asthma ruled out. In the adjusted analyses, a confirmed

25 diagnosis was associated with higher direct healthcare costs (Relative Ratio [RR] $=1.60,95 \% \mathrm{CI}$

26 1.14-2.22), increased rate of specialist visits $(\mathrm{RR}=2.41,95 \% \mathrm{CI} 1.05-5.40)$ and reliever

27 medication use $(\mathrm{RR}=1.62,95 \% \mathrm{CI} 1.09-2.35)$, but not primary care physician visits $(\mathrm{p}=0.10)$ or

28 controller medication use $(\mathrm{p}=0.11)$.

29 Conclusions: A quarter of individuals with a physician diagnosis of asthma did not have asthma 
30 after objective re-evaluation. These participants still consumed a significant amount of asthma-

31 related healthcare resources. The population-level economic burden of asthma overdiagnosis

32 could be substantial.

33

34 Strengths and limitations of this study:

35 - Participants were recruited through random sampling of the general population in the

36 province of British Columbia.

37 - Asthma diagnosis was confirmed or ruled out using sequential guideline-recommended

38 objective airway tests.

39 - Healthcare resource use was self-reported, potential recall bias may have led to reduced

$40 \quad$ accuracy.

41 - The study was unable to evaluate the indirect costs of overdiagnosis or the cost-savings

42 from correcting the diagnosis.

43 - The generalizability of the results may be limited by regional differences in medical costs

$44 \quad$ and practices.

45

46 Keywords: Asthma, Costs, Misdiagnosis, Observational Studies, Overdiagnosis 


\section{INTRODUCTION}

48 Over 300 million people worldwide have been diagnosed with asthma.[1] Patients with asthma

49 experience symptoms of wheezing, shortness of breath, chest tightness, and cough.[2] These

50 symptoms, and periods of intensified disease activity referred to as exacerbations, impose a

51 significant burden on healthcare resources and reduce patient quality of life.[3] A Canadian study

52 estimated the excess direct medical costs of asthma at \$1,058 (2013 Canadian dollars) per

53 person-year.[4] The majority (74\%) of asthma-attributed costs were due to medication use.[4]

55 Multiple evidence-based guidelines recommend confirming a diagnosis of asthma with objective

56 testing for reversible airflow limitation or increased airway hyper responsiveness.[2,5] Despite

57 these recommendations, previous studies suggest that in the community, asthma is diagnosed

58 solely based on symptom history in over half of the cases.[6,7] The underuse of spirometry has

59 been documented in Canada,[8] the United States,[9] and Europe.[10] A recent population-based

60 study found that one in three patients with physician-diagnosed asthma did not meet the

61 guideline-recommended spirometric criteria for asthma diagnosis and could have their

62 medications safely stopped.[11] We refer to this group of patients as 'overdiagnosed'.[12] These

63 overdiagnosed patients are likely to be imposing costs on the healthcare system due to treatment

64 for a condition that does not exist, and may be experiencing symptoms of an underlying illnesses

65 that is not being treated.[11] By some estimates, there are over 785,000 overdiagnosed asthma

66 patients in Canada alone.[13]

67

68 In response to these findings, some authors have called for population-based screening or case

69 finding to re-evaluate previous diagnoses of asthma.[11,13] Assessing the value of these 
70 programs requires precise estimates of the burden of overdiagnosed asthma. To the best of our

71 knowledge, estimates of the cost differences between overdiagnosed and confirmed cases of

72 asthma currently do not exist. A previous study of the costs of overdiagnosed asthma was limited

73 to assessing the potential asthma-related cost savings that a secondary screening program could

74 provide.[13] Characterizing the patterns of healthcare resource use among patients in whom

75 asthma can be ruled out can help identify opportunities for re-evaluation and inform initiatives to

76 improve asthma diagnosis in the community.

78 We used a longitudinal population-based cohort of individuals with physician-diagnosed asthma

79 to address this important evidence gap. Our primary objective was to compare total direct

80 asthma-related healthcare costs in patients with a confirmed diagnosis of asthma versus patients

81 in whom a diagnosis of asthma was ruled out using objective testing. Our secondary objectives

82 were to characterize differences in healthcare resource use in terms of the 1) number of

83 outpatient physician visits, and the 2) type and amount of asthma medication use.

\section{METHODS}

\section{Study Design and Sample}

88 We used longitudinal data from the Economic Burden of Asthma study (University of British

89 Columbia Human Ethics \#H10-01542), which has been previously described.[14,15] In

90 summary, individuals with a self-reported physician diagnosis of asthma and at least one asthma-

91 related healthcare encounter in the past 5 years were eligible. Participants were recruited through

92 random-digit dialling of both landlines and mobile phones in the census subdivisions of 
93 Vancouver and Central Okanagan (populations of 603,502 and 179,839 in 2011, respectively) of

94 BC, Canada.[16] Between 2010 and 2012, 618 participants were recruited, evaluated at baseline,

95 and followed for 12 months with visits at 3-month intervals. We included 345 participants who

96 were $\geq 12$ years of age at baseline and successfully completed a bronchodilator reversibility or

97 methacholine challenge test at the end of follow-up.

98

99 Outcomes

100 Participants reported their asthma-related primary care and specialist physician visits,

101 hospitalizations, emergency department visits, and current medication use at each study visit

102 with a recall period of 3 months. The primary outcome was total asthma-related direct healthcare

103 costs per patient over the one-year follow-up period. Total direct healthcare costs were

104 comprised of all outpatient and inpatient encounters, and medication costs incurred by the

105 patient. Cost categories were described by calculating the percentage of total average cost

106 constituted by each category. The methods used for calculating costs are detailed

107 elsewhere.[17,18] Briefly, costs for each patient were determined by multiplying self-reported

108 resource use quantities by unit cost of each resource. We determined the average unit cost of an

109 asthma-related physician visit (specialist vs. primary care) and hospitalization for the years 2008-

1102012 using International Classification of Diseases codes from provincial healthcare

111 administrative data.[18] Medication unit costs were determined by linking Drug Identification

112 Numbers of participant-reported asthma medications to the Provincial Drug Master Plan

113 database.[19] Cost per dose was estimated using the lowest price equivalent of the medication.

114 All costs were adjusted to 2017 Canadian Dollars[20] and the analysis was conducted from the

115 perspective of the Canadian healthcare system. 
117 Secondary outcomes were the number of asthma-related outpatient physician visits and use of

118 asthma medications. We did not evaluate asthma-related emergency department visits or

119 hospitalizations as a separate outcome due to the low frequency of these events $(\mathrm{N}=14)$. The

120 number of outpatient physician visits over one-year of follow-up was assessed separately by

121 physician type (primary care or specialist). Medication use was captured using the questionnaire

122 shown in Figure E1, and medications were classified into controller or reliever using a reference

123 list (Table E1). In general, controller medications are those with anti-inflammatory effects

124 (namely inhaled corticosteroids and leukotriene receptor antagonists), while reliever medications

125 are those that are used on as-needed basis for temporary symptom relief (namely short-acting

126 beta agonists). Medication use was determined by calculating the Medication Possession Ratio

127 (MPR) separately for controller and reliever medications. MPR represents the proportion of days

128 in which medications were available to the participant over the follow-up period.[21]

\section{Exposure: Objective confirmation of asthma}

131 Participants underwent an objective assessment of asthma at the final visit. The diagnostic

132 algorithm for asthma is shown in Figure 1 and consisted of both bronchodilator reversibility and

133 methacholine challenge tests implemented in a stepwise fashion. Spirometry was performed by a

134 trained technician using a regularly calibrated spirometer. Reversible airflow obstruction was

135 defined as a $\geq 12 \%$ increase in Forced Expiratory Volume in 1 second (FEV 1$) 15$ minutes after

136 administration of $200 \mathrm{mcg}$ of salbutamol via pressurized metered dose inhaler and a spacer

137 device.[22] Participants who did not meet the criteria for reversible airflow obstruction returned

138 within one week to undergo a methacholine challenge test. Participants who did not meet the 
139 criteria for asthma diagnosis at the first methacholine challenge test had their controller

140 medications tapered and discontinued by a respirologist before returning for a second

141 methacholine challenge test. A diagnosis of asthma was ruled out if FEV 1 decreased by $<20 \%$

142 following the administration of $16 \mathrm{mg} / \mathrm{mL}$ of methacholine[23] in both methacholine challenge

143 tests. Participants had a 'confirmed asthma diagnosis' if they met the criteria for asthma at the

144 bronchodilator reversibility test or either methacholine challenge test.

\section{Statistical Analysis}

149 All statistical analyses were performed in R version 3.5.0.[24] We considered a two-tailed p150 value of $<0.05$ as statistically significant.

152 We constructed separate generalized linear regression models (negative binomial distribution, $153 \log$ link) for the primary and secondary outcomes. This resulted in five separate models for the

154 following outcomes: annual asthma-related (1) direct healthcare costs, (2) number of primary

155 care physician visits, (3) number of specialist physician visits, (4) controller MPR, and (5)

156 reliever MPR. All models included the objective diagnosis of asthma (confirmed vs. ruled out) as

157 the exposure. Costs and MPR values (as a percentage) were rounded to the nearest integer value.

158 Models were adjusted for participant tobacco smoking history (ever smoked vs. never smoked),

159 ethnicity (Caucasian vs. non-Caucasian), age, sex, education (postsecondary vs. no), income

160 (annual household income $\geq \$ 70,000 \mathrm{vs.} \mathrm{less),} \mathrm{and} \mathrm{third-party} \mathrm{insurance} \mathrm{coverage} \mathrm{for} \mathrm{medications}$

161 (coverage vs. no), all self-reported at baseline with a 12-month recall period. The resulting 
162 regression coefficients were exponentiated to create effect estimates on the relative scale (rate

163 ratio $[R R])$. Asthma severity was not adjusted for due to the high likelihood that there is a causal

164 relationship between severe asthma and a confirmed diagnosis of asthma.[11]

165

166

167 RESULTS

168 Sample Characteristics

169 The cohort selection procedure is illustrated in Figure 2. From the Economic Burden of Asthma

170 cohort (618 participants), we excluded 86 participants who were $<12$ years of age, 153 in whom

171 a methacholine challenge was contraindicated $(\mathrm{N}=29)$, refused $(\mathrm{N}=112)$, or could not be

172 completed for other reasons $(\mathrm{N}=14)$, and 34 participants who were lost to follow-up. The final

173 study cohort included 345 participants who underwent an objective diagnostic test for asthma at

174 the final visit (12 months after baseline). Their characteristics are shown in Table 1. 212 (61.4\%)

175 participants were female, and the mean age at baseline was 48.9 (standard deviation [SD] 17.8)

176 years. A diagnosis of asthma was confirmed in 259 (75.1\%) participants: $138(53.3 \%)$ by

177 bronchodilator reversibility test, $98(37.8 \%)$ following the first methacholine challenge test, and

$17823(8.9 \%)$ following the second methacholine challenge test. Asthma was ruled out in 86

$179(24.9 \%)$ participants following negative results on all bronchodilator reversibility and

180 methacholine challenge tests.

184 Table 1. Sample characteristics 


\begin{tabular}{|c|c|c|c|c|}
\hline Characteristic & $\begin{array}{c}\text { Total } \\
(n=345)\end{array}$ & $\begin{array}{c}\text { Asthma } \\
\text { confirmed } \\
(n=259) \\
\end{array}$ & $\begin{array}{c}\text { Asthma } \\
\text { ruled out } \\
(\mathrm{n}=\mathbf{8 6}) \\
\end{array}$ & $p$-value ${ }^{\S}$ \\
\hline \multicolumn{5}{|c|}{ Variables evaluated at baseline } \\
\hline $\begin{array}{c}\text { Female } \\
\mathrm{n}(\%)\end{array}$ & $212(61.4)$ & $156(60.2)$ & $56(65.1)$ & 0.50 \\
\hline $\begin{array}{c}\text { Age, } \\
\text { mean }(\mathrm{SD}) \\
\end{array}$ & $48.9(17.8)$ & $49.3(18.1)$ & $47.7(17.2)$ & 0.46 \\
\hline $\begin{array}{c}\text { Ever smoked (vs. never smoked), } \\
\mathrm{n}(\%)\end{array}$ & $93(27.0)$ & $71(27.4)$ & $22(25.6)$ & 0.85 \\
\hline $\begin{array}{c}\text { Caucasian (vs. non-Caucasian), } \\
\mathrm{n}(\%)\end{array}$ & $267(77.4)$ & $206(79.5)$ & $61(70.9)$ & 0.13 \\
\hline $\begin{array}{c}\text { High income (vs. low income })^{\dagger}, \\
\mathrm{n}(\%)\end{array}$ & $183(53.0)$ & $137(52.9)$ & $46(53.5)$ & $>0.99$ \\
\hline $\begin{array}{c}\text { Health insurance (full coverage } \\
\text { vs. not full coverage })^{\ddagger} \\
n(\%)\end{array}$ & $69(20.0)$ & $52(20.1)$ & $17(19.8)$ & $>0.99$ \\
\hline $\begin{array}{l}\text { Higher education (postsecondary } \\
\text { vs. no postsecondary), } \\
\mathrm{n}(\%)\end{array}$ & $242(70.1)$ & $178(68.7)$ & $64(74.4)$ & 0.39 \\
\hline \multicolumn{5}{|c|}{ Variables evaluated during follow-up } \\
\hline $\begin{array}{l}\text { Total direct healthcare costs, } \\
\text { mean (SD) }\end{array}$ & $\begin{array}{c}\$ 450.5 \\
(\$ 629.2) \\
\end{array}$ & $\begin{array}{r}\$ 497.9 \\
(\$ 677.9) \\
\end{array}$ & $\begin{array}{c}\$ 307.7 \\
(\$ 424.1) \\
\end{array}$ & $<0.01^{*}$ \\
\hline $\begin{array}{c}\text { Primary care physician visits, } \\
\text { mean (SD) }\end{array}$ & $2.2(4.1)$ & $2.1(2.8)$ & $2.7(6.6)$ & 0.85 \\
\hline $\begin{array}{c}\text { Specialist physician visits, } \\
\text { mean (SD) }\end{array}$ & $0.5(1.9)$ & $0.6(2.2)$ & $0.2(0.8)$ & $0.02^{*}$ \\
\hline $\begin{array}{l}\text { MPR of controller medications, } \\
\text { mean (SD) }\end{array}$ & $69.7 \%(74.4)$ & $74.9 \%(76.4)$ & $54.0 \%(65.8)$ & $0.02^{*}$ \\
\hline $\begin{array}{c}\text { MPR of reliever medications, } \\
\text { mean (SD) }\end{array}$ & $28.4 \%(33.2)$ & $31.3 \%(34.3)$ & $19.5 \%(28.0)$ & $<0.01^{*}$ \\
\hline
\end{tabular}

MPR: Medication Possession Ratio

* Significant at 0.05 level

${ }^{\dagger}$ High income defined as household income $\geq \$ 70,000$

$\$$ Full coverage defined as all drug and physician services paid for through third-party coverage

$\S$ p-values for the absolute difference between the 'asthma confirmed' and 'asthma ruled out'

groups were determined using a Student t-test for continuous variables and Chi-squared test for categorical variables 


\section{Total Direct Healthcare Costs}

188 Average annual direct healthcare costs over one year was \$497.9 (SD \$677.9) in participants

189 with confirmed asthma, and \$307.7 (SD \$424.1) in those with asthma ruled out. There was a

190 significant difference in average annual direct healthcare costs $(\$ 190.2)$ between exposure

191 groups in the unadjusted analysis $(\mathrm{p}<0.01$, Table 1$)$. Average annual direct healthcare costs were

1921.6 times $(95 \% \mathrm{CI} 1.14-2.22, \mathrm{p}<0.01)$ higher in participants with confirmed asthma after

193 adjustment for participant smoking history, ethnicity, age, sex, education, income, and insurance

194 coverage (Figure 3). Medications comprised the greatest proportion of total costs in both

195 correctly diagnosed and overdiagnosed individuals (56.4\% and 47.6\%, respectively, Table 2).

Table 2. Asthma-related healthcare cost categories over one year of follow-up.

\begin{tabular}{|c|c|c|}
\hline Cost Category & $\begin{array}{c}\text { Cost per patient } \\
\text { mean (SD) }\end{array}$ & $\begin{array}{c}\text { Percentage of total cost per } \\
\text { patient }\end{array}$ \\
\hline Medications & \multicolumn{2}{|c|}{ Asthma ruled out } \\
\hline Primary care physician visits & $\$ 146.39(\$ 239.94)$ & $47.6 \%$ \\
\hline Specialist physician visits & $\$ 27.87(\$ 101.37)$ & $38.4 \%$ \\
\hline Hospitalization & $\$ 0(\$ 0)$ & $9.1 \%$ \\
\hline Emergency visits & $\$ 15.23(\$ 69.07)$ & $0.0 \%$ \\
\hline TOTAL & $\$ 307.70(\$ 422.23)$ & $4.9 \%$ \\
\hline Medications & \multicolumn{2}{|c|}{ Asthma confirmed } \\
\hline Primary care physician visits & $\$ 90.98(\$ 125.66)$ & $56.4 \%$ \\
\hline Specialist physician visits & $\$ 73.11(\$ 257.61)$ & $18.3 \%$ \\
\hline Hospitalization & $\$ 39.24(\$ 362.65)$ & $14.7 \%$ \\
\hline Emergency visits & $\$ 13.91(\$ 72.07)$ & $7.9 \%$ \\
\hline TOTAL & $\$ 497.90(\$ 676.89)$ & $100.8 \%$ \\
\hline
\end{tabular}




\section{Outpatient Physician Visits}

203 The total number of annual primary care physician visits was similar between participants with

204 confirmed asthma and those with asthma ruled out (2.1 vs. 2.7 visits, $\mathrm{p}=0.85$, Table 1$)$. However,

205 participants with confirmed asthma visited specialist physicians more often than those who had

206 asthma ruled out ( 0.6 vs. 0.2 visits per year, $\mathrm{p}=0.02)$. The adjusted analysis also indicated no

207 significant difference in the number of primary care physician visits between participants with

208 confirmed asthma and those with asthma ruled out $(\mathrm{p}=0.10$, Figure 3$)$. Confirmed asthma was

209 associated with 2.41 times $(95 \%$ CI $1.05-5.40, \mathrm{p}=0.03)$ more specialist visits than when an

210 asthma diagnosis could be ruled out.

212 Medication Usage

213 Participants with a confirmed diagnosis of asthma used controller medications for a greater

214 proportion of follow-up time than those with asthma ruled out (MPR of $74.9 \%$ vs. 54.0\%,

$215 \mathrm{p}=0.02)$, and similarly for reliever medications $(31.3 \%$ vs. $19.5 \%, \mathrm{p}<0.01$, Table 1$)$. This

216 difference persisted in the adjusted analysis for reliever medications but not for controller

217 medications ( $\mathrm{p}=0.11$, Figure 3). The MPR for reliever medications was 1.62 times higher

218 (95\%CI 1.09-2.35, $\mathrm{p}=0.01)$ among participants with confirmed asthma than those with asthma

219 ruled out. 


\section{DISCUSSION}

223 In this study, we used objective testing to confirm the diagnosis of asthma in a population-based

224 sample of patients with a self-reported physician diagnosis of asthma. We found that asthma

225 could be ruled out in $25 \%$ of cases after negative spirometry and two negative methacholine

226 challenge tests. This proportion is in line with the $28-33 \%$ rate of overdiagnosis reported in

227 previous Canadian studies.[11,13,25] We compared asthma-related direct healthcare costs

228 between participants with confirmed asthma versus those with asthma ruled out. Although total

229 direct costs were higher in participants with a confirmed diagnosis of asthma, the costs of

230 overdiagnosed asthma remained substantial. The average direct asthma-related healthcare costs

231 for a participant with overdiagnosed asthma was \$308 over 12 months, which was \$190 lower

232 than for participants with a confirmed diagnosis. This difference in costs remained statistically

233 significant after controlling for confounding variables that could affect both the exposure and

234 outcomes but are likely not on the causal pathway between them.

236 Participants with overdiagnosed asthma visited specialists less frequently than those with

237 confirmed asthma, but they visited primary care physicians as frequently. These participants may

238 have scheduled a similar number of annual primary care visits for routine monitoring of 'asthma'

239 activity or to fill new prescriptions. Conversely, participants with confirmed asthma may have

240 had exacerbations of their asthma symptoms that required referral to a specialist physician to

241 improve asthma control.[26] Across both groups, participants visited primary care physicians

242 more frequently than specialist physicians; $79 \%$ of our sample visited a primary care physician at

243 least once during follow-up. The high frequency of these visits, and their low cost compared to

244 specialist consultations,[13] suggests that primary care visits may provide effective opportunities 
245 for re-evaluating previous asthma diagnoses.

247 On average, overdiagnosed participants possessed controller medications for over half of follow-

248 up time, and reliever medications for 20 percent of follow-up time. Reliever medications are

249 typically used as needed while controller medications are used daily.[2,5] In comparison to

250 participants with confirmed asthma, and after adjusting for potential confounders, participants

251 with asthma ruled out tended to use similar levels of controller medications and less reliever

252 medications. This difference may be due to a lower symptom burden in overdiagnosed

253 participants, which led to self-adjustment of reliever medication use.[27] Conversely,

254 overdiagnosed individuals may have used controller medications as prescribed.

256 Although inhaled medications for asthma are generally safe, the use of asthma medications

257 among overdiagnosed patients puts them at risk of net harm due to medication side effects.[28]

258 These patients are also incurring additional healthcare expenditure without therapeutic benefit.

259 Further, asthma medications may have masked symptoms of a serious underlying illness and

260 resulted in a delay in the diagnosis and treatment of the correct disease. We did not evaluate the

261 true underlying condition in this sample, but a similar study by Aaron et al.[11] found that two

262 out of 213 patients had subglottic stenosis, which was treated as asthma for a number of years

263 before the correct diagnosis was identified during the study. If other health conditions were

264 responsible for the overdiagnosis of asthma, their costs have implications for the burden of

265 overdiagnosed asthma. For example, if ruling out an asthma diagnosis results in the correct

266 alternative diagnosis, the proper management of the underlying condition could confer further

267 cost benefits. 
269 To our knowledge, this is the first study to examine the costs associated with overdiagnosed

270 asthma. Our findings have important population-level implications. Given an estimate of

271 approximately 785,000 individuals with overdiagnosed asthma in Canada,[13] and an annual

272 direct healthcare cost of $\$ 308$ per patient, the estimated cost of overdiagnosed asthma in Canada

273 is \$242 million per year (2017 Canadian dollars). A previous study reported an average cost of

$274 \$ 263$ per patient (2009 Canadian dollars) for additional physician visits to reassess an asthma

275 diagnosis. This resulted in an average lifetime cost savings of \$351 per patient screened,[13]

276 primarily due to the avoided costs of asthma medications. Our results suggest that additional

277 screening to correct an overdiagnosis of asthma would save asthma-related costs in the first year,

278 with savings compounding in subsequent years. Savings could be reallocated to the management

279 of individuals with a true diagnosis of asthma, especially those with severe asthma who could

280 pursue novel but expensive treatments, or to identify and treat the underlying diseases of

281 overdiagnosed patients.[29] Given the prevalence and costs of overdiagnosis, our findings

282 highlight the need for routine objective testing to confirm all new and existing diagnoses of

283 asthma.[2,5]

285 There are several strengths to this study. We used a population-based random sample of patients

286 with a physician diagnosis of asthma, meaning our results are likely to be representative of

287 routine healthcare use in the general asthma population. We used longitudinal healthcare

288 utilization data, which compared to cross-sectional data, likely provides a less-biased estimate of

289 the average costs accrued by an individual. Finally, we evaluated airway reversibility using 
290 objective testing following the recommendations of international guidelines.[2,5] This allowed

291 us to evaluate the costs of evidence-based best practices.

292

293 Our study has several limitations. We did not include indirect costs in our analysis, and we were

294 therefore unable to consider the societal costs of overdiagnosis. Previous studies suggest that the

295 cost of productivity loss in this sample is substantial.[15] Physician visits and medication use

296 were self-reported with a recall period of 3 months. It is possible that recall bias reduced the

297 accuracy of our measurements. Our algorithm for ruling out asthma involved one less

298 methacholine challenge test than in some previous studies, $[11,13,30]$ which makes it slightly less

299 rigorous. It is possible that this led to a higher rate of overdiagnosis in our study, however, over

$30090 \%$ of diagnoses were confirmed by the second visit in previous studies.[13,25] We excluded

301 participants in whom an objective diagnostic test was contraindicated due to asthma-related

302 reasons, which may have resulted in lower representation of participants with more severe

303 disease and therefore higher resource utilization. Finally, we only assessed asthma-related costs;

304 we were unable to determine the costs of the true underlying condition or the benefit of

305 correcting the diagnosis.

CONCLUSIONS

309 In this population-based sample, one in four participants with a physician diagnosis of asthma

310 had their diagnosis ruled out upon objective airflow reversibility testing. Patients with

311 overdiagnosed asthma consumed a substantial amount of asthma-related healthcare resources,

312 although less than those with confirmed asthma. The extent of overdiagnosed asthma in Canada 
313 and other countries, and its associated healthcare resource costs, suggests that the population-

314 level burden of overdiagnosed asthma is high. Future studies should evaluate the cost-

315 effectiveness of systematic screening or case detection initiatives for re-evaluating previous

316 diagnoses of asthma.

317

318

319 Acknowledgements: We would like to thank all members of the Economic Burden of Asthma

320 study team. JMF and MS conceived, designed, and conducted the Economic Burden of Asthma

321 study. MS formulated the current study idea and MS, KMJ and BCN designed the study. BCN

322 performed all data analyses and wrote the first draft of the manuscript. AS and KMJ provided

323 guidance on the statistical analysis. All authors critically commented on the manuscript and

324 approved the final version. KMJ is the guarantor of the manuscript.

326 Competing Interests: BCN, AS, JMF, and KMJ have no conflicts to declare. MS has received

327 honorarium for unrelated consultancy work from GSK Canada and GSK global.

329 Funding: This study was funded by Collaborative Innovative Research Fund, an arm's length,

330 investigator initiated, peer-reviewed grant from GSK Canada. The funders had no role in study

331 design, data collection and analysis, or preparation of the manuscript. 


\section{REFERENCES}

3331 Braman SS. The Global Burden of Asthma. Chest 2006;130:4S-12S.

334 doi:10.1378/CHEST.130.1_SUPPL.4S

3352 Global Initiative for Asthma (GINA). Global Strategy for Asthma Management and

336 Prevention, 2018. https://ginasthma.org/2018-gina-report-global-strategy-for-asthma-

337 management-and-prevention (accessed: August 23, 2018).

3383 Bousquet J, Bousquet PJ, Godard P, et al. The public health implications of asthma. Bull

$339 \quad$ World Health Organ 2005;83:548-54.

3404 Chen W, Lynd LD, FitzGerald JM, et al. Excess medical costs in patients with asthma and 341 the role of comorbidity. Eur Respir J 2016;48:1584-92. doi:10.1183/13993003.01141$342 \quad 2016$

3435 National Institutes of Health. Expert Panel Report 3: Guidelines for the Diagnosis and 344 Management of Asthma Full Report 2007. Natl Hear Lung, Blood Inst 2007.

3456 Jin R, Choi BC, Chan BT, et al. Physician asthma management practices in Canada. Can $346 \quad$ Respir J 2000;7:456-65. doi:10.1155/2000/587151

3477 Sharpe HM, Sin DD, Andrews EM, et al. Alberta Strategy to Help Manage Asthma 348 (ASTHMA): a provincial initiative to improve outcomes for individuals with asthma. $349 \quad$ Healthc $Q$ 2004;7:4,55-60.

3508 Gershon AS, Victor JC, Guan J, et al. Pulmonary function testing in the diagnosis of 351 asthma: a population study. Chest 2012;141:1190-6. doi:10.1378/chest.11-0831

3529 Sokol KC, Sharma G, Lin Y-L, et al. Choosing wisely: adherence by physicians to 353 recommended use of spirometry in the diagnosis and management of adult asthma. Am J $354 \quad$ Med 2015;128:502-8. doi:10.1016/j.amjmed.2014.12.006

35510 Heffler E, Crimi C, Mancuso S, et al. Misdiagnosis of asthma and COPD and underuse of $356 \quad$ spirometry in primary care unselected patients. Respir Med 2018;142:48-52. 
doi:10.1016/j.rmed.2018.07.015

35811 Aaron SD, Vandemheen KL, FitzGerald JM, et al. Reevaluation of diagnosis in adults

359 with physician-diagnosed asthma. JAMA - J Am Med Assoc 2017;317:269-79. doi:10.1001/jama.2016.19627

12 Aaron SD, Boulet LP, Reddel HK, et al. Underdiagnosis and Overdiagnosis of Asthma. Am J Respir Crit Care Med 2018;198:1012-20. doi:10.1164/rccm.201804-0682CI

13 Pakhale S, Sumner A, Coyle D, et al. (Correcting) misdiagnoses of asthma: a cost effectiveness analysis. BMC Pulm Med 2011;11:27. doi:10.1186/1471-2466-11-27

Chen W, FitzGerald JM, Rousseau R, et al. Complementary and alternative asthma treatments and their association with asthma control: a population-based study. BMJ Open 2013;3:e003360.

16 [dataset] British Columbia Statistics. Open Data from the Census - BC Stats. Census Div. Regional Dist. Census Subdiv. Municipalities, RDEAs, Indian Reserv. 2011. http://www.bcstats.gov.bc.ca/StatisticsBySubject/Census/OpenData.aspx (accessed 15 May 2018). guideline-based asthma symptom control: A population-based study. Allergy Eur $J$ Allergy Clin Immunol Published Online First: 2016. doi:10.1111/all.12803 
resources/pharmacare/health-industry-professionals/downloadable-drug-data-files (accessed 19 Jul 2018).

[dataset] Statistics Canada. Table 18-10-0005-01 Consumer Price Index, annual average, not seasonally adjusted 2018 . https:/www150.statcan.gc.ca/t1/tb11/en/tv.action?pid=1810000501 (accessed 8 August 2018). doi: $10.1002 /$ pds. 1230

Boulet LP, Becker A, Berube D, et al. Canadian Asthma Consensus Report, 1999. Canadian Asthma Consensus Group. CMAJ 1999;161:S1-61.

Andrade SE, Kahler KH, Frech F, et al. Methods for evaluation of medication adherence Care Med 2000;161:309-29. doi:10.1164/ajrccm.161.1.ats11-99

R Core Team. R: A Language and Environment for Statistical Computing. 2018.

Luks VP, Vandemheen KL, Aaron SD. Confirmation of asthma in an era of overdiagnosis. Eur Respir J 2010;36:255 LP - 260.

26 Schatz M, Rachelefsky G, Krishnan JA. Follow-up after acute asthma episodes: what improves future outcomes? Proc Am Thorac Soc 2009;6:386-93. doi:10.1513/pats.P09ST6

Horne R, Weinman J. Self-regulation and Self-management in Asthma: Exploring The Role of Illness Perceptions and Treatment Beliefs in Explaining Non-adherence to Preventer Medication. Psychol Health 2002;17:17-32. doi:10.1080/08870440290001502

BJ L. Systemic adverse effects of inhaled corticosteroid therapy: A systematic review and meta-analysis. Arch Intern Med 1999;159:941-55. 

the family physician. Can Respir J 2004;11:349-53. doi:10.1155/2004/914865

$41030 \quad$ Aaron SD, Vandemheen KL, Boulet L-P, et al. Overdiagnosis of asthma in obese and

411 nonobese adults. C Can Med Assoc J 2008;179:1121-31. doi:10.1503/cmaj.081332 


\section{FIGURE LEGENDS}

Figure 1. Algorithm for confirming or ruling out a diagnosis of asthma.

$\mathrm{FEV}_{1}$; Forced Expiratory Volume in 1 second, $\mathrm{PC}_{20}$; Provocative concentration of methacholine needed to produce a $20 \%$ fall in $\mathrm{FEV}_{1}$ from baseline

Figure 2. Sample selection procedure.

Figure 3. Relative Ratios for the adjusted differences in asthma-related a) total direct healthcare costs, b) primary care physician visits, c) specialist physician visits, d) controller medication use, and e) reliever medication use over one year of follow-up between patients with confirmed asthma and those with asthma ruled out.

* Significant at 0.05 level 


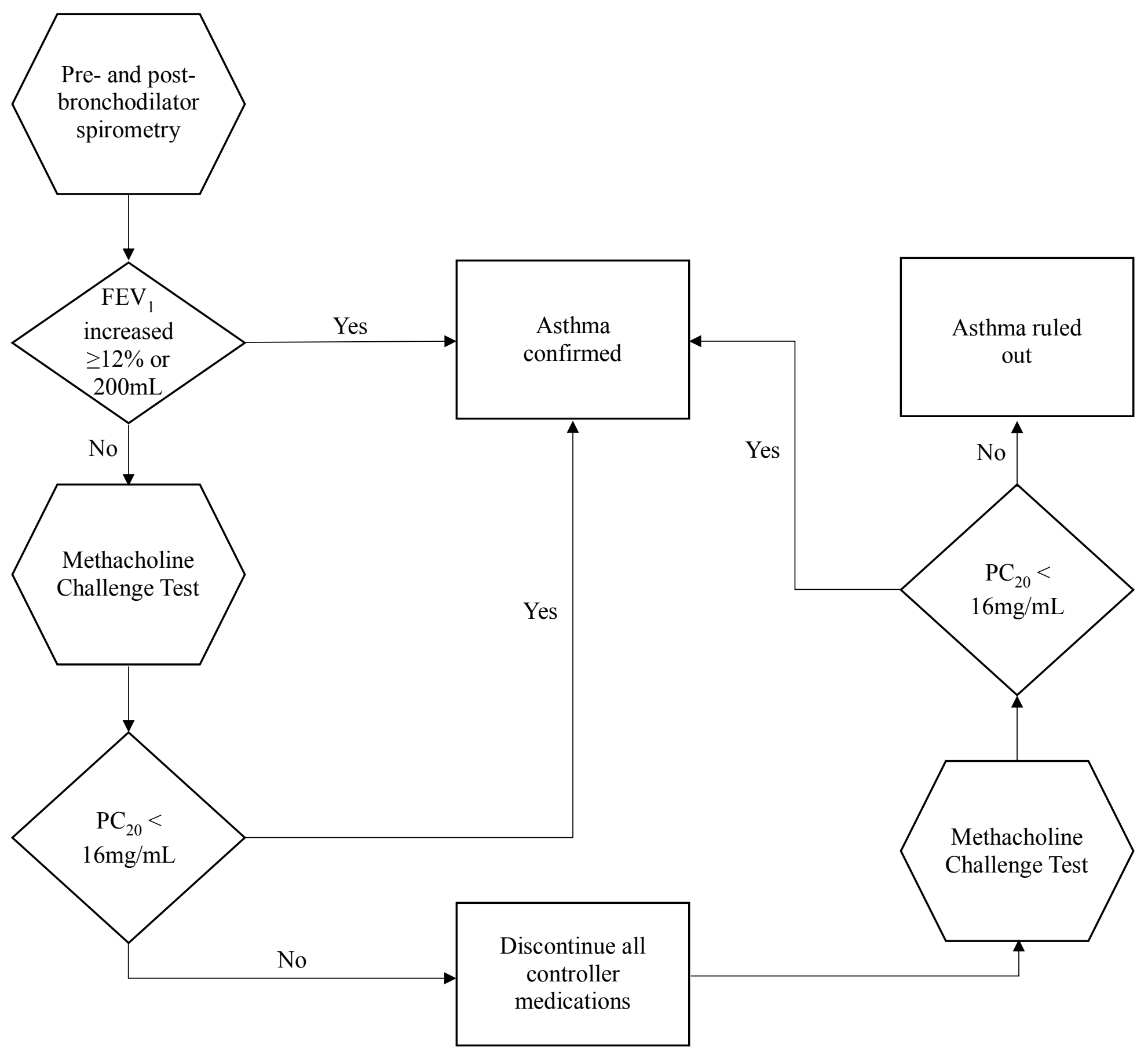


50,572 Individuals contacted through random digit dialing

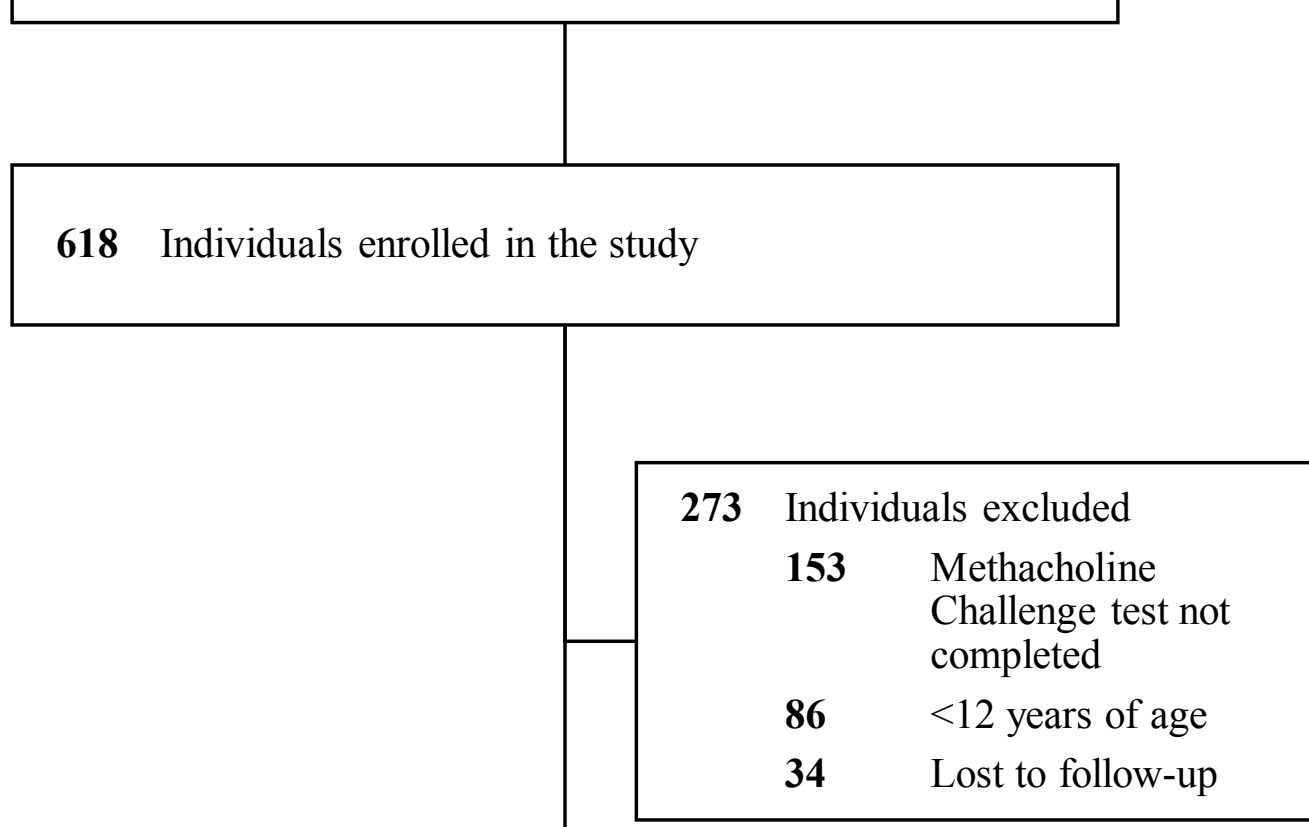

259 Individuals with asthma diagnosis confirmed

138 + Bronchodilator Reversibility test

86 Individuals with asthma diagnosis ruled out

121 + Methacholine Challenge test 
a) Total Direct Healthcare Costs

Asthma Confirmed (vs. ruled out)*

Relative Ratio $95 \% \mathrm{CI}$

Caucasian (vs. non-Caucasian) Age (10-year increase) Female (vs. male)

Higher Education (postsecondary vs. no postsecondary)

High Income (vs. low income)

High Insurance (full coverage vs. no)
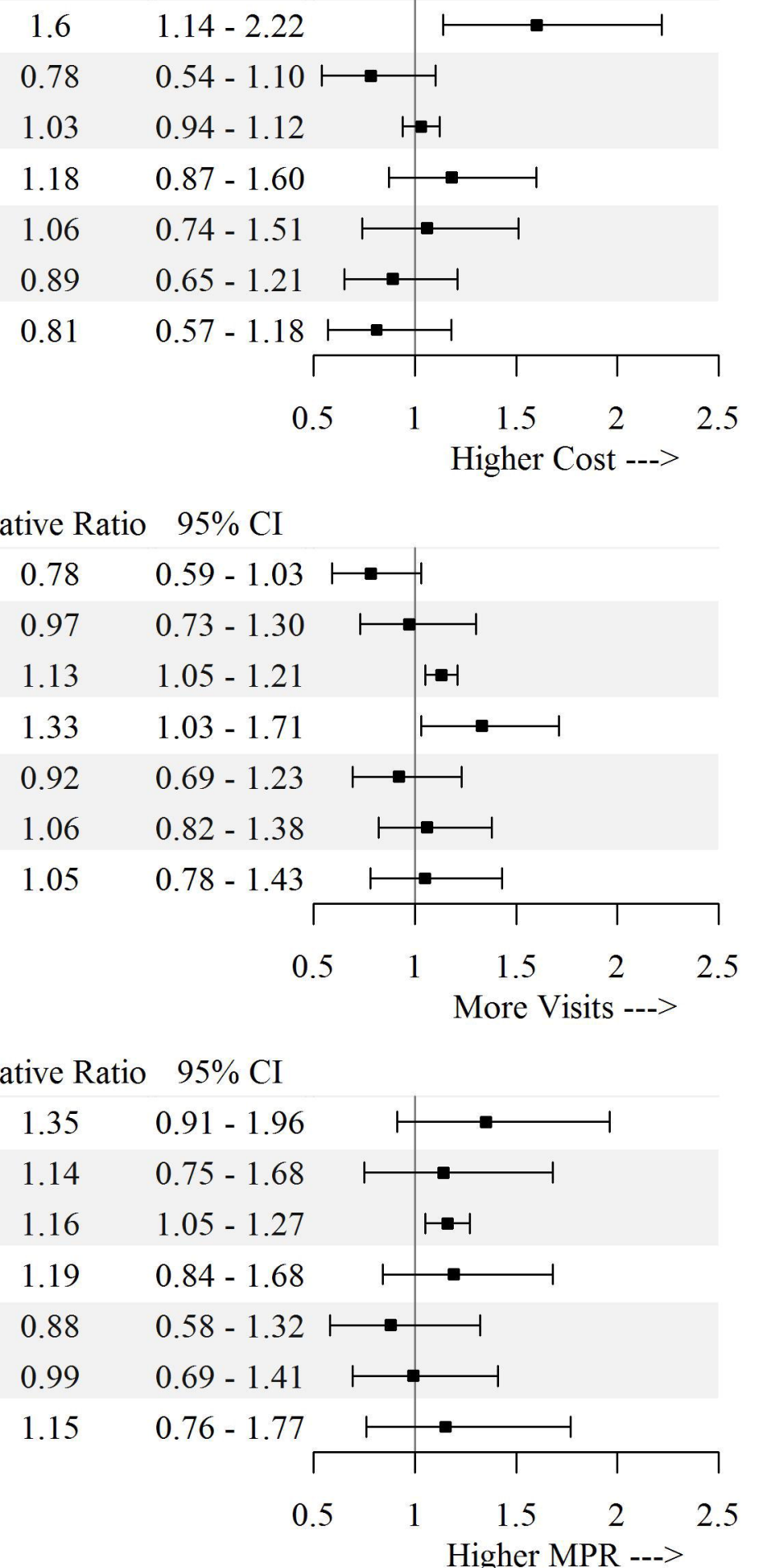

b) Primary Care Physician Visits

Asthma Confirmed (vs ruled out)

Caucasian (vs non-Caucasian)

Age (10-year increase)*

Female (vs. male)*

Relative Ratio 95\% CI

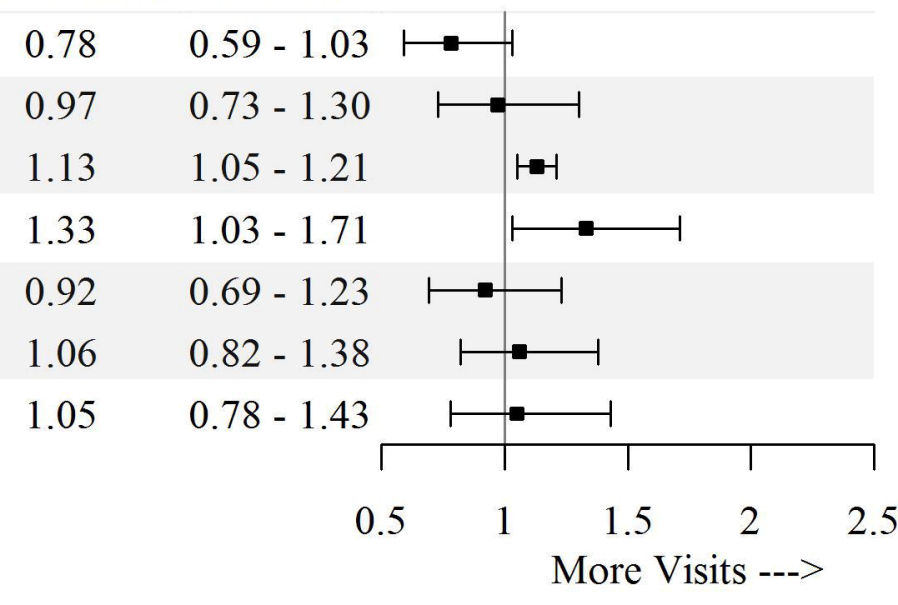

d) Controller Medication Use Asthma Confirmed (vs ruled out)

Relative Ratio $95 \%$ CI

Caucasian (vs non-Caucasian) Age (10-year increase)* Female (vs. male) c) Specialist Physician Visits Asthma Confirmed (vs ruled out)* Caucasian (vs non-Caucasian) Age (10-year increase) Female (vs. male)

Higher Education (postsecondary vs. no postsecondary)

High Income (vs. low income)

High Insurance (full coverage vs. no)*

e) Reliever Medication Use Asthma Confirmed (vs ruled out)*

Caucasian (vs non-Caucasian)

Age (10-year increase)

Female (vs. male)*

Higher Education (postsecondary vs. no postsecondary)

High Income (vs. low income)

High Insurance (full coverage vs. no)
Relative Ratio $95 \% \mathrm{CI}$

$1.06-5.42$

$0.22-1.06$

$0.74-1.10$

$0.87-3.76$

$1.28-6.62$

$0.38-1.71 \longleftrightarrow$

$0.15-0.94 \longleftarrow$

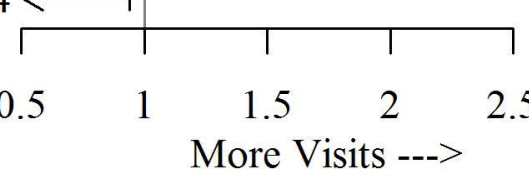

Higher Education (postsecondary vs. no postsecondary)

$$
\text { High Income (vs. low income) }
$$

High Insurance (full coverage vs. no)

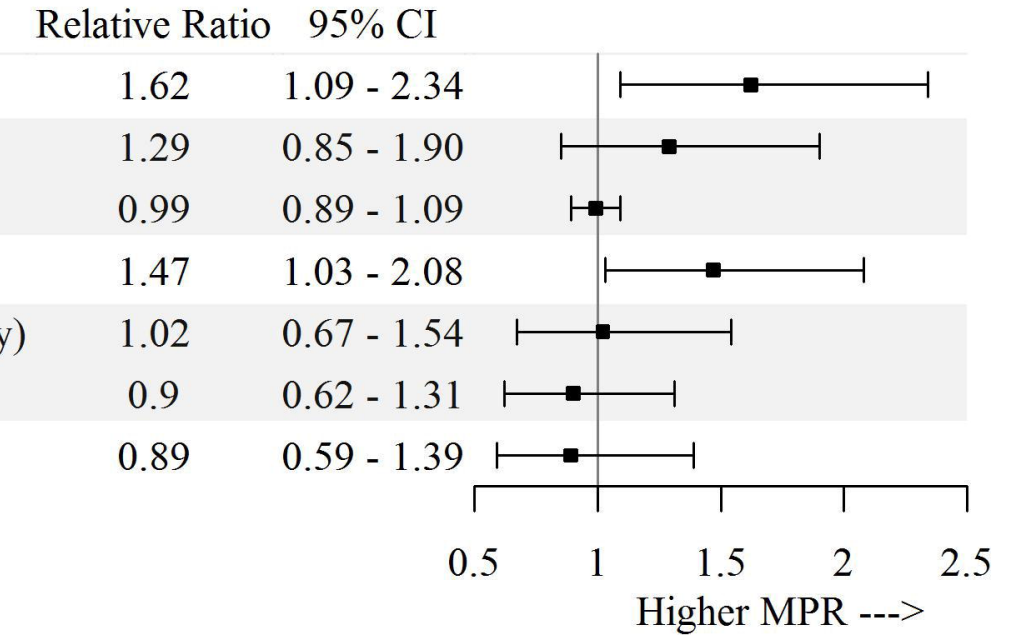

